\title{
Magnetic fields in White Dwarfs and their direct progenitors
}

\author{
Stefan Jordan \\ Astronomisches Rechen-Institut, Zentrum für Astronomie, Mönchhofstr. 12-14, \\ D-69120 Heidelberg, Germany \\ email: jordan@ari.uni-heidelberg.de
}

\begin{abstract}
The paper provides an overview on the results of the analyses of spectro-polarimetric observations of white dwarfs, subdwarfs, and central stars of planetary nebulae. It will also discuss the question of the origin of the magnetic fields in white dwarfs.
\end{abstract}

Keywords. White Dwarfs - magnetic fields - subdwarfs - Planetary Nebulae

\section{Introduction and history}

Blackett (1947) predicted that very strong magnetic fields $(\approx 1 \mathrm{MG})$ could exist in white dwarfs if the magnetic moment of a star is proportional to its angular momentum, which he assumed to be conserved during the stellar evolution and the collapse. This is, however, probably not the case since most isolated white dwarfs seem to be relatively slow rotators $(v \lesssim 40 \mathrm{~km} / \mathrm{sec}$, see Karl et al. 2005 and references thererein), although a few exceptions from this rule exist (e.g. REJ 0317-853 with a rotational period of 725 sec, Barstow et al. 1995); if angular momentum were completely conserved during the evolution we would expect the white dwarf remnant to have $\mathrm{v}_{\text {rot }} \approx 10,000 \mathrm{~km} / \mathrm{sec}$.

Another possibility was proposed by Ginzburg (1964) and Woltjer (1964). They argued that if the magnetic flux, which is proportional to $B R^{2}$, is conserved during evolution and collapse, very strong magnetic fields can be reached in degenerate stars. A main sequence star with a radius $R \approx 10^{11} \mathrm{~cm}$ and a surface magnetic field of $1-10 \mathrm{kG}$ can therefore become a white dwarf $\left(R \approx 10^{9} \mathrm{~cm}\right)$ with a magnetic field strength of $10^{7}-10^{8} \mathrm{G}$.

The search for magnetic white dwarfs began in 1970 when Preston looked for quadratic Zeeman shifts in the spectra of DA white dwarfs. Due to the extremely strongly Stark broadened Balmer lines and the limited spectral resolution he was only able to place upper limits of about 0.5 MG for the magnetic fields in several white dwarfs. A rather sensitive method to detect magnetic fields in white dwarfs is the measurement of circular polarization. Kemp (1970) detected circular polarization of several percent in Grw $+70^{\circ} 8247$, an object that was known for its rather shallow and unidentified "Minkowski bands" (Minkowski 1938). Although his derived value for the magnetic field strength was wrong this proved the existence of a magnetic field in this white dwarf. Nevertheless, all attempts to identify the Minkowski bands with various atoms or molecules in magnetic fields of a few MG failed.

Even for the simplest atoms, hydrogen and helium, accurate calculations for the line components did not exist at that time for field strengths above 20-100 MG (Kemic 1974a,b); only for extremely intense fields $\left(10^{9}-10^{10} \mathrm{G}\right)$ data were available again Garstang (1977), but none of the predicted line positions were in agreement with the wavelengths of the Grw $+70^{\circ} 8247$ features. For this reason Angel (1979) proposed that the star must possess a field strength above $100 \mathrm{MG}$ (but below the intense-field regime). 

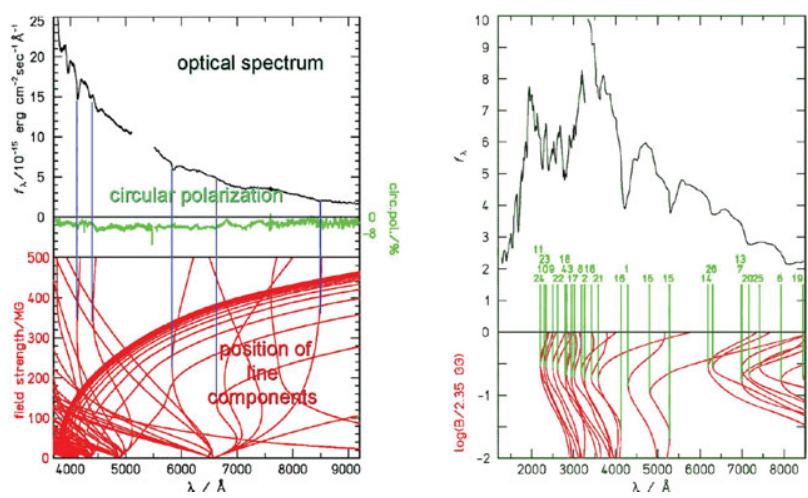

Figure 1. Spectrum and circular polarisation of the famous magnetic white dwarf Grw $+70^{\circ} 8247$ (left) can the identification of the spectral features with stationary line components; the dipole field strength is $\approx 320 \mathrm{MG}$ (Jordan 1992). On the right hand side, the spectrum of GD 229 is compared to stationary line components of neutral helium (Jordan et al. 1998).

For hydrogen the intermediate-field gap has been closed more than twenty years ago with calculations of energy level shifts and transition probabilities for bound-bound transitions by groups in Tübingen and Baton Rouge (Forster et al. 1984; Rösner et al. 1984; Henry \& O'Connell 1984).

\section{Stationary line components}

Since the magnetic field on the surface of a white dwarf normally is not homogeneous but e.g. better described by a magnetic dipole or more complicated field geometries, the variation of the field strengths from the pole to the equator (a factor of two for a pure dipole field) smears out most of the absorption lines; this explains why the spectral features on Grw $+70^{\circ} 8247$ are so shallow. However, a few of the line components become stationary, i.e. their wavelengths go through maxima or minima as functions of the magnetic field strength. These stationary components are visible in the spectra of magnetic white dwarfs despite a considerable variation of the field strengths.

It was a great confirmation for the correctness of the theoretical calculations that indeed the unidentified features in the optical and UV spectrum of Grw $+70^{\circ} 8247$ could be attributed to stationary components of hydrogen (see Fig. 1) in fields between about 150 and $500 \mathrm{MG}$ (Greenstein 1984; Greenstein et al. 1985).

For helium reliable atomic data for arbitrary magnetic field strengths became available only in the late nineties (see Jordan et al. 1998, 2001) when the existence of helium could be proven (Jordan et al. 1998).

Table 1: List of all known MWDs, extending the one by Kawka et al. (2007), containing the name of the object, the effective temperature, the chemical composition, the magnetic field strength (usually the dipole field strength), the rotational period. References are provided only if not provided by Kawka et al. (2007). For uncertainties of the values please refer to the original papers.

\begin{tabular}{lrllcl}
\hline \hline Object & $T_{\text {eff }} / \mathrm{kK}$ & Comp. & $B / \mathrm{MG}$ & $P_{\text {rot }}$ & References \\
\hline SDSS J000555.91-100213.4 & 29000 & $\mathrm{He} / \mathrm{C}$ & $?$ & $\ldots$ & \\
LHS 1038 & 6540 & $\mathrm{H}$ & $\lesssim 0.2$ & $>2 \mathrm{~h}$ & \\
LHS 1044 & 6010 & $\mathrm{H}$ & 16.7 & $\ldots$ & \\
SDSS J001742.44+004137.4 & 15000 & $\mathrm{He}$ & 8.30 & $\ldots$ & \\
SDSS J002129.00+150223.7 & $7000 \mathrm{H}$ & 530.69 & $\ldots$ & Külebi et al. $(08)$ \\
SDSS J004248.19+001955.3 & $11000 \mathrm{H}$ & 2.00 & $\ldots$ & Külebi et al. $(08)$ \\
Feige 7 & 20000 & $\mathrm{H} / \mathrm{He}$ & 35.00 & $12 \mathrm{~m}$ & \\
\hline
\end{tabular}


Table 1: continued.

\begin{tabular}{|c|c|c|c|c|c|}
\hline Object & $\overline{T_{\text {eff }} / \mathrm{kK}}$ & Comp. & $B / \mathrm{MG}$ & $P_{\text {rot }}$ & References \\
\hline SDSS J014245.37+131546.4 & 15000 & $\mathrm{He}$ & 4.00 & $\cdots$ & \\
\hline SDSS J015748.15+003315.1 & $\sim 6000$ & $\begin{array}{l}\mathrm{He} \\
(\mathrm{DZ})\end{array}$ & 3.70 & $\cdots$ & \\
\hline MWD 0159-032 & 26000 & $\mathrm{H}$ & 6.00 & $\ldots$ & \\
\hline SDSS J021116.34+003128.5 & 9000 & $\mathrm{H}$ & 341.31 & .. & Külebi et al. (08) \\
\hline SDSS J021148.22+211548.2 & 12000 & $\mathrm{H}$ & 168.20 & $\ldots$ & Külebi et al. (08) \\
\hline SDSS J023609.40-080823.9 & 10000 & $\begin{array}{l}\mathrm{H} \\
\text { (DQA) }\end{array}$ & 5.00 & $\cdots$ & \\
\hline HE $0236-2656$ & 6500 & $\mathrm{He}$ & $?$ & $\ldots$ & \\
\hline HE $0241-155$ & 12000 & $\mathrm{H}$ & 200 & $\ldots$ & Reimers et al. (04) \\
\hline KPD $0253+5052$ & 15000 & $\mathrm{H}$ & 13.5 & $\cdots$ & \\
\hline LHS 5064 & 6680 & $\mathrm{H}$ & $\sim 0.1$ & $\ldots$ & \\
\hline SDSS J030407.40-002541.7 & 15000 & $\mathrm{H}$ & 10.95 & $\cdots$ & Külebi et al. (08) \\
\hline MWD 0307-428 & 25000 & $\mathrm{H}$ & 10.00 & $\ldots$ & \\
\hline SDSS J031824.19+422651.0 & 10500 & $\mathrm{H}$ & 10.12 & $\ldots$ & Külebi et al. (08) \\
\hline REJ J0317-853 & 33000 & $\mathrm{H}$ & $>180$ & $725 \mathrm{~s}$ & \\
\hline KUV $03292+0035$ & 26500 & $\mathrm{H}$ & 12.10 & $\cdots$ & \\
\hline HE 0330-0002 & 6500 & $\mathrm{He}$ & $?$ & $\cdots$ & \\
\hline SDSS J033145.69+004517.0 & 15500 & $\mathrm{H}$ & 13.13 & $\cdots$ & Külebi et al. (08) \\
\hline SDSS J034308.18-064127.3 & 13000 & $\mathrm{H}$ & 19.78 & $\ldots$ & Külebi et al. (08) \\
\hline SDSS J034511.11+003444.3 & 8000 & $\mathrm{H}$ & 1.96 & $\ldots$ & Külebi et al. (08) \\
\hline 40 Eri B & 16490 & $\mathrm{H}$ & 0.0023 & $\cdots$ & \\
\hline BPM 3523 & 23450 & $\mathrm{H}$ & 0.00428 & $\cdots$ & \\
\hline LHS 1734 & 5300 & $\mathrm{H}$ & 7.3 & $\ldots$ & \\
\hline G $99-37$ & 6070 & $\mathrm{C}_{2} / \mathrm{CH}$ & 7.5 & $4.1 \mathrm{~h}$ & Berd. et al. (07) \\
\hline G $99-47$ & 5790 & $\mathrm{H}$ & 20 & $0.97 \mathrm{~h}$ & \\
\hline EUVE J0616-649 & 50000 & $\mathrm{H}$ & 14.80 & $\cdots$ & \\
\hline GD 77 & 14870 & $\mathrm{H}$ & 1.2 & $\cdots$ & \\
\hline G $234-4$ & 4500 & $\mathrm{H}$ & 0.0396 & $\cdots$ & \\
\hline SDSS J074850.48+301944.8 & 22000 & $\mathrm{H}$ & 6.75 & $\cdots$ & Külebi et al. (08) \\
\hline SDSS J075234.96+172525.0 & 9000 & $\mathrm{H}$ & 10.30 & $\ldots$ & Külebi et al. (08) \\
\hline SDSS J075819.57+354443.7 & 22000 & $\mathrm{H}$ & 26.40 & $\cdots$ & Külebi et al. (08) \\
\hline G $111-49$ & 8500 & $\mathrm{H}$ & 220 & $\cdots$ & \\
\hline SDSS J080440.35+182731.0 & 11000 & $\mathrm{H}$ & 48.47 & $\cdots$ & Külebi et al. (08) \\
\hline SDSS J080502.29+215320.5 & 28000 & $\mathrm{H}$ & 6.11 & $\cdots$ & Külebi et al. (08) \\
\hline SDSS J080743.33+393829.2 & 13000 & $\mathrm{H}$ & 65.75 & $\ldots$ & Külebi et al. (08) \\
\hline SDSS J080938.10+373053.8 & 14000 & $\mathrm{H}$ & 39.74 & $\cdots$ & Külebi et al. (08) \\
\hline SDSS J081648.71+041223.5 & 11500 & $\mathrm{H}$ & 7.35 & $\cdots$ & Külebi et al. (08) \\
\hline GD 90 & 14000 & $\mathrm{H}$ & 9.00 & $\cdots$ & \\
\hline EUVE J0823-254 & 43200 & $\mathrm{H}$ & $2.8-3.5$ & $\cdots$ & \\
\hline SDSS J082835.82+293448.7 & 19500 & $\mathrm{H}$ & 33.40 & $\ldots$ & Külebi et al. (08) \\
\hline EG 61 & 17100 & $\mathrm{H}$ & $\sim 3$ & $\cdots$ & \\
\hline SDSS J084008.50+271242.7 & 12250 & $\mathrm{H}$ & 3.38 & $\cdots$ & Külebi et al. (08) \\
\hline SDSS J084155.74+022350.6 & 7000 & $\mathrm{H}$ & 5.00 & $\cdots$ & Külebi et al. (08) \\
\hline SDSS J083945.56+200015.7 & 15000 & $\mathrm{H}$ & 3.38 & $\cdots$ & \\
\hline SDSS J084716.21+484220.4 & 19000 & $\mathrm{H}$ & $\sim 3$ & $\cdots$ & \\
\hline
\end{tabular}


Table 1: continued.

\begin{tabular}{|c|c|c|c|c|c|}
\hline Object & $T_{\text {eff }} / \mathrm{kK}$ & Comp. & $B / \mathrm{MG}$ & $P_{\text {rot }}$ & References \\
\hline SDSS J085106.12+120157.8 & 11000 & $\mathrm{H}$ & 2.03 & $\cdots$ & Külebi et al. (08) \\
\hline LB 8915 & 24000 & $\mathrm{H} / \mathrm{He}$ & $0.75-1.0$ & $\cdots$ & \\
\hline SDSS J085523.87+164059.0 & 15500 & $\mathrm{H}$ & 12.23 & $\cdots$ & Külebi et al. (08) \\
\hline SDSS J085830.85+412635.1 & 7000 & $\mathrm{H}$ & 3.38 & $\ldots$ & Külebi et al. (08) \\
\hline SDSS J090632.66+080716.0 & 17000 & $\mathrm{H}$ & 10.00 & ... & \\
\hline SDSS J090746.84+353821.5 & 16500 & $\mathrm{H}$ & 22.40 & $\cdots$ & Külebi et al. (08) \\
\hline SDSS J091124.68+420255.9 & 10250 & $\mathrm{H}$ & 35.20 & $\cdots$ & Külebi et al. (08) \\
\hline SDSS J091437.40+054453.3 & 17000 & $\mathrm{H}$ & 9.16 & $\cdots$ & Külebi et al. (08) \\
\hline G $195-19$ & 7160 & $\mathrm{He}$ & $\sim 100$ & $1.3 \mathrm{~d}$ & \\
\hline SDSS J091833.32+205536.9 & 14000 & $\mathrm{H}$ & 2.04 & $\ldots$ & Külebi et al. (08) \\
\hline SDSS J092527.47+011328.7 & 10000 & $\mathrm{H}$ & 2.04 & $\cdots$ & Külebi et al. (08) \\
\hline SDSS J093313.14+005135.4 & $\cdots$ & $\begin{array}{l}\mathrm{He} \\
\left(\mathrm{C}_{2} \mathrm{H}\right) ?\end{array}$ & $?$ & $\cdots$ & \\
\hline SDSS J093356.40+102215.7 & 8500 & $\mathrm{H}$ & 2.11 & $\cdots$ & Külebi et al. (08) \\
\hline SDSS J093447.90+503312.2 & 8900 & $\mathrm{H}$ & 9.50 & $\ldots$ & Külebi et al. (08) \\
\hline SDSS J094458.92+453901.2 & 15500 & $\mathrm{H}$ & 15.91 & $\cdots$ & \\
\hline LB 11146 & 16000 & $\mathrm{H}$ & 670.00 & $\cdots$ & \\
\hline SDSS J095442.91+091354.4 & $\cdots$ & DQ & $?$ & $\cdots$ & \\
\hline SDSS J100005.67+015859.2 & 9000 & $\mathrm{H}$ & 19.74 & $\cdots$ & Külebi et al. (08) \\
\hline SDSS J100356.32+053825.6 & 23000 & $\mathrm{H}$ & 10.13 & $\cdots$ & Külebi et al. (08) \\
\hline SDSS J100657.51+303338.1 & 10000 & $\mathrm{H}$ & 1.00 & .. & Külebi et al. (08) \\
\hline SDSS J100715.55+123709.5 & 18000 & $\mathrm{H}$ & 5.41 & $\cdots$ & Külebi et al. (08) \\
\hline LHS 2229 & 4600 & $\begin{array}{l}\mathrm{He} \\
\left(\mathrm{C}_{2} \mathrm{H}\right)\end{array}$ & $\sim 100$ & $\cdots$ & \\
\hline SDSS J101529.62+090703.8 & 7200 & $\mathrm{H}$ & 4.09 & $\cdots$ & Külebi et al. (08) \\
\hline SDSS J101618.37+040920.6 & 10000 & $\mathrm{H}$ & 7.94 & $\ldots$ & Külebi et al. (08) \\
\hline PG $1015+014$ & 14000 & $\mathrm{H}$ & 70.00 & $99 \mathrm{~m}$ & Euchner et al. (06) \\
\hline GD 116 & 16000 & $\mathrm{H}$ & 65 & $\cdots$ & \\
\hline SDSS J102239.06+194904.3 & 9000 & $\mathrm{H}$ & 2.94 & $\cdots$ & Külebi et al. (08) \\
\hline LHS 2273 & 7160 & $\mathrm{H}$ & 18.00 & $\cdots$ & \\
\hline PG $1031+234$ & $\sim 15000$ & $\mathrm{H}$ & $\lesssim 1000$ & $3.4 \mathrm{~h}$ & \\
\hline SDSS J103655.38+652252.0 & $\cdots$ & DQ & 0.17 & $\cdots$ & \\
\hline LP $790-29$ & 7800 & $\mathrm{He}$ & 50.00 & $26 \mathrm{y} ?$ & \\
\hline HE 1043-0502 & $\sim 15000$ & $\mathrm{He}$ & $\sim 820$ & $\cdots$ & \\
\hline HE 1045-0908 & 10000 & $\mathrm{H}$ & 16.00 & $2.7 \mathrm{~h}$ & \\
\hline SDSS J105404.38+593333.3 & 9500 & $\mathrm{H}$ & 17.63 & $\cdots$ & Külebi et al. (08) \\
\hline SDSS J105628.49+652313.5 & 16500 & $\mathrm{H}$ & 29.27 & $\cdots$ & Külebi et al. (08) \\
\hline LTT 4099 & 15280 & $\mathrm{H}$ & 0.0039 & $\cdots$ & \\
\hline SDSS J111010.50+600141.4 & 30000 & $\mathrm{H}$ & 6.37 & $\cdots$ & Külebi et al. (08) \\
\hline SDSS J111341.33+014641.7 & $\cdots$ & He ? & $?$ & $\cdots$ & \\
\hline SDSS J111812.67+095241.4 & 10500 & $\mathrm{H}$ & 3.38 & $\cdots$ & Külebi et al. (08) \\
\hline SDSS J112257.10+322327.8 & 12500 & $\mathrm{H}$ & 11.38 & $\cdots$ & Külebi et al. (08) \\
\hline SDSS J112852.88-010540.8 & 11000 & $\mathrm{H}$ & 2.00 & $\cdots$ & Külebi et al. (08) \\
\hline SDSS J112924.74+493931.9 & 10000 & $\mathrm{H}$ & 5.31 & $\cdots$ & \\
\hline SDSS J113357.66+515204.8 & 22000 & $\mathrm{H}$ & 8.64 & $\cdots$ & Külebi et al. (08) \\
\hline SDSS J113756.50+574022.4 & 7800 & $\mathrm{H}$ & 5.00 & $\cdots$ & Külebi et al. (08) \\
\hline
\end{tabular}


Table 1: continued.

\begin{tabular}{|c|c|c|c|c|c|}
\hline Object & $T_{\text {eff }} / \mathrm{kK}$ & Comp. & $B / \mathrm{MG}$ & $P_{\text {rot }}$ & References \\
\hline LBQS 1136-0132 & 10500 & $\mathrm{H}$ & 22.71 & $\cdots$ & \\
\hline SDSS J114006.37+611008.2 & 13500 & $\mathrm{H}$ & 50.19 & $\cdots$ & Külebi et al. (08) \\
\hline SDSS J114829.00+482731.2 & 27500 & $\mathrm{H}$ & 32.47 & $\cdots$ & Külebi et al. (08) \\
\hline SDSS J115418.14+011711.4 & 1125 & $\mathrm{H}$ & 33.47 & $\ldots$ & Külebi et al. (08) \\
\hline SDSS J115917.39+613914.3 & 23000 & $\mathrm{H}$ & 20.10 & $\ldots$ & Külebi et al. (08) \\
\hline SDSS J120150.10+614257.0 & 10500 & $\mathrm{H}$ & 11.35 & $\cdots$ & Külebi et al. (08) \\
\hline SDSS J120609.80+081323.7 & 13000 & $\mathrm{H}$ & 760.63 & $\cdots$ & Külebi et al. (08) \\
\hline SDSS J120728.96+440731.6 & 16750 & $\mathrm{H}$ & 2.03 & $\cdots$ & Külebi et al. (08) \\
\hline SDSS J121209.31+013627.7 & 10000 & $\mathrm{H}$ & 10.12 & $\ldots$ & Külebi et al. (08) \\
\hline HE $1211-1707$ & $\sim 12000$ & $\mathrm{He}$ & 50.00 & $\sim 2 \mathrm{~h}$ & \\
\hline LHS 2534 & 6000 & $\begin{array}{l}\mathrm{He} \\
(\mathrm{DZ})\end{array}$ & 1.92 & $\cdots$ & \\
\hline SDSS J121635.37-002656.2 & 20000 & $\mathrm{H}$ & 59.70 & $\ldots$ & Külebi et al. (08) \\
\hline SDSS J122209.44+001534.0 & 20000 & $\mathrm{H}$ & 14.70 & $\ldots$ & Külebi et al. (08) \\
\hline PG $1220+234$ & 26540 & $\mathrm{H}$ & 3.00 & $\ldots$ & \\
\hline SDSS J122249.14+481133.1 & 9000 & $\mathrm{H}$ & 8.05 & $\cdots$ & Külebi et al. (08) \\
\hline SDSS J122401.48+415551.9 & 9500 & $\mathrm{H}$ & 22.36 & $\cdots$ & Külebi et al. (08) \\
\hline SDSS J123414.11+124829.6 & 8200 & $\mathrm{H}$ & 4.32 & $\cdots$ & Külebi et al. (08) \\
\hline SDSS J124806.38+410427.2 & 7000 & $\mathrm{H}$ & 7.03 & $\cdots$ & Külebi et al. (08) \\
\hline SDSS J124851.31-022924.7 & 13500 & $\mathrm{H}$ & 7.36 & $\ldots$ & Külebi et al. (08) \\
\hline SDSS J125044.42+154957.4 & 10000 & $\mathrm{H}$ & 20.71 & $\ldots$ & Külebi et al. (08) \\
\hline SDSS J125416.01+561204.7 & 13250 & $\mathrm{H}$ & 38.86 & $\cdots$ & Külebi et al. (08) \\
\hline HS $1254+3440$ & 15000 & $\mathrm{H}$ & 9.5 & $\cdots$ & \\
\hline SDSS J125434.65+371000.1 & 10000 & $\mathrm{H}$ & 4.10 & $\cdots$ & Külebi et al. (08) \\
\hline SDSS J125715.54+341439.3 & 8500 & $\mathrm{H}$ & 11.45 & $\ldots$ & Külebi et al. (08) \\
\hline G $256-7$ & $\sim 56000$ & $\mathrm{H}$ & 4.9 & $\ldots$ & \\
\hline PG $1312+098$ & $\sim 20000$ & $\mathrm{H}$ & 10.00 & $5.4 \mathrm{~h}$ & \\
\hline SDSS J132002.48+131901.6 & 14750 & $\mathrm{H}$ & 2.02 & $\cdots$ & Külebi et al. (08) \\
\hline SDSS J132858.20+590851.0 & 25000 & $\begin{array}{l}\mathrm{H} \\
\text { (DQA) }\end{array}$ & 18.00 & $\cdots$ & \\
\hline G165-7 & 6440 & $\begin{array}{l}\mathrm{He} \\
(\mathrm{DZ})\end{array}$ & 0.65 & $\ldots$ & \\
\hline G $62-46$ & 6040 & $\mathrm{H}$ & 7.36 & $\cdots$ & \\
\hline SDSS J133359.86+001654.8 & $\ldots$ & $\begin{array}{l}\mathrm{He} \\
\left(\mathrm{C}_{2} \mathrm{H}\right) ?\end{array}$ & $?$ & $\ldots$ & \\
\hline SDSS J133340.34+640627.4 & 13500 & $\mathrm{H}$ & 10.71 & $\ldots$ & Külebi et al. (08) \\
\hline SDSS J134043.10+654349.2 & 15000 & $\mathrm{H}$ & 4.32 & $\cdots$ & Külebi et al. (08) \\
\hline SDSS J134820.79+381017.2 & 35000 & $\mathrm{H}$ & 13.65 & $\cdots$ & Külebi et al. (08) \\
\hline SBS $1349+5434$ & 11000 & $\mathrm{H}$ & 760 & $\cdots$ & \\
\hline LP 907-037 & 9520 & $\mathrm{H}$ & $\lesssim 0.3$ & $\cdots$ & \\
\hline SDSS J140716.66+495613.7 & 20000 & $\mathrm{H}$ & 12.49 & $\ldots$ & Külebi et al. (08) \\
\hline SDSS J141906.19+254356.5 & 9000 & $\mathrm{H}$ & 2.03 & $\cdots$ & Külebi et al. (08) \\
\hline SDSS J142625.71+575218.3 & 19830 & $\begin{array}{l}\mathrm{He} / \mathrm{C}_{2} \\
\text { (DQ) }\end{array}$ & $\sim 1.2$ & $\cdots$ & Dufour et al. $(08)$ \\
\hline SDSS J142703.40+372110.5 & 19000 & $\mathrm{H}$ & 27.04 & $\cdots$ & Külebi et al. (08) \\
\hline SDSS J143019.05+281100.8 & 9000 & $\mathrm{H}$ & 9.34 & $\cdots$ & Külebi et al. (08) \\
\hline
\end{tabular}


Table 1: continued.

\begin{tabular}{|c|c|c|c|c|c|}
\hline Object & $T_{\text {eff }} / \mathrm{kK}$ & Comp. & $B / \mathrm{MG}$ & $P_{\text {rot }}$ & References \\
\hline SDSS J143218.26+430126.7 & 24000 & $\mathrm{H}$ & 2.04 & $\cdots$ & Külebi et al. (08) \\
\hline SDSS J143235.46+454852.5 & 16750 & $\mathrm{H}$ & 12.29 & $\cdots$ & Külebi et al. (08) \\
\hline EUVE J1439+750 & $>20000$ & $\mathrm{H}$ & $14-16$ & $\cdots$ & \\
\hline SDSS J144614.00+590216.7 & 12500 & $\mathrm{H}$ & 4.42 & $\cdots$ & Külebi et al. (08) \\
\hline SDSS J145415.01+432149.5 & 11500 & $\mathrm{H}$ & 2.35 & $\ldots$ & Külebi et al. (08) \\
\hline GD 175 & 6990 & $\mathrm{H}$ & 2.30 & $\ldots$ & \\
\hline SDSS J150813.20+394504.9 & 17000 & $\mathrm{H}$ & 13.23 & $\cdots$ & Külebi et al. (08) \\
\hline SDSS J151130.20+422023.0 & 9750 & $\mathrm{H}$ & 22.40 & $\cdots$ & Külebi et al. (08) \\
\hline SDSS J151745.19+610543.6 & 9500 & $\mathrm{H}$ & 13.98 & $\cdots$ & Külebi et al. (08) \\
\hline GD 185 & 18620 & $\mathrm{H}$ & 0.035 & $\cdots$ & \\
\hline PG $1533-057$ & 20000 & $\mathrm{H}$ & 31 & $\cdots$ & \\
\hline SDSS J153532.25+421305.6 & 18500 & $\mathrm{H}$ & 5.27 & $\cdots$ & Külebi et al. (08) \\
\hline SDSS J153829.29+530604.6 & 13500 & $\mathrm{H}$ & 13.99 & $\cdots$ & Külebi et al. (08) \\
\hline SDSS J154213.48+034800.4 & 8500 & $\mathrm{H}$ & 8.35 & $\cdots$ & Külebi et al. (08) \\
\hline SDSS J160437.36+490809.2 & 9000 & $\mathrm{H}$ & 59.51 & $\cdots$ & Külebi et al. (08) \\
\hline GD 356 & 7510 & $\mathrm{He}$ & 13.00 & $0.1 \mathrm{~d}$ & \\
\hline SDSS J164357.02+240201.3 & 16500 & $\mathrm{H}$ & 2.00 & $\cdots$ & Külebi et al. (08) \\
\hline SDSS J164703.24+370910.3 & 16250 & $\mathrm{H}$ & 2.10 & $\cdots$ & Külebi et al. (08) \\
\hline SDSS J165029.91+341125.5 & 9750 & $\mathrm{H}$ & 3.38 & $\cdots$ & Külebi et al. (08) \\
\hline SDSS J165203.68+352815.8 & 11500 & $\mathrm{H}$ & 7.37 & $\cdots$ & Külebi et al. (08) \\
\hline PG $1658+440$ & 30510 & $\mathrm{H}$ & 2.3 & $\ldots$ & \\
\hline SDSS J170400.01+321328.7 & 23000 & $\mathrm{H}$ & 50.11 & $\cdots$ & Külebi et al. (08) \\
\hline NLTT 44447 & 6260 & $\mathrm{H}$ & 1.30 & $\cdots$ & \\
\hline SDSS J171556.29+600643.9 & 13500 & $\mathrm{H}$ & 2.03 & $\cdots$ & Külebi et al. (08) \\
\hline SDSS J172045.37+561214.9 & 22500 & $\mathrm{H}$ & 19.79 & $\cdots$ & Külebi et al. (08) \\
\hline SDSS J172329.14+540755.8 & 16500 & $\mathrm{H}$ & 32.85 & $\ldots$ & Külebi et al. (08) \\
\hline SDSS J172932.48+563204.1 & 10500 & $\mathrm{H}$ & 27.26 & $\cdots$ & Külebi et al. (08) \\
\hline BPM 25114 & $\sim 20000$ & $\mathrm{H}$ & 36.00 & $2.8 \mathrm{~d}$ & \\
\hline G $240-72$ & 5590 & $\mathrm{He}$ & $\gtrsim 100$ & $\gtrsim 20 \mathrm{y}$ & \\
\hline G $183-35$ & 6500 & $\mathrm{H}$ & $\sim 14$ & $\sim 50 \mathrm{~m} ?$ & \\
\hline G $141-2$ & 6340 & $\mathrm{H}$ & $\sim 3$ & $\cdots$ & \\
\hline G $227-35$ & 6280 & $\mathrm{H}$ & 175 & $\cdots$ & \\
\hline Grw $+70^{\circ} 8247$ & 16000 & $\mathrm{H}$ & 320 & $\cdots$ & \\
\hline G $92-40$ & 7920 & $\mathrm{H}$ & $\gtrsim 0.1$ & $1.4 \mathrm{~d}$ & \\
\hline GD 229 & 18000 & $\mathrm{He}$ & 700 & $\cdots$ & Jordan et al. (98) \\
\hline LTT 7987 & 15360 & $\mathrm{H}$ & 0.001 & $\cdots$ & Jordan et al. (07) \\
\hline SDSS J202501.10+131025.6 & 17000 & $\mathrm{H}$ & 10.10 & $\cdots$ & Külebi et al. (08) \\
\hline SDSS J204626.15-071037.0 & 8000 & $\mathrm{H}$ & 2.03 & $\cdots$ & Külebi et al. (08) \\
\hline SDSS J205233.52-001610.7 & 19000 & $\mathrm{H}$ & 13.42 & $\cdots$ & Külebi et al. (08) \\
\hline L $24-52$ & 10200 & $\mathrm{H}$ & $0.043 ?$ & $\cdots$ & \\
\hline SDSS J214900.87+004842.8 & 11000 & $\mathrm{H}$ & 10.09 & $\cdots$ & Külebi et al. (08) \\
\hline SDSS J214930.74-072812.0 & 22000 & $\mathrm{H}$ & 44.71 & $\cdots$ & Külebi et al. (08) \\
\hline SDSS J215135.00+003140.5 & 9000 & $\mathrm{H}$ & $\sim 300$ & $\cdots$ & Külebi et al. (08) \\
\hline SDSS J215148.31+125525.5 & 14000 & $\mathrm{H}$ & 20.76 & $\cdots$ & Külebi et al. (08) \\
\hline SDSS J220435.05+001242.9 & 22000 & $\mathrm{H}$ & 1.02 & $\cdots$ & Külebi et al. (08) \\
\hline SDSS J221828.59-000012.2 & 15500 & $\mathrm{H}$ & 225.00 & $\cdots$ & \\
\hline
\end{tabular}


Table 1: continued.

\begin{tabular}{lrllll}
\hline \hline Object & $T_{\text {eff }} / \mathrm{kK}$ & Comp. & $B / \mathrm{MG}$ & $P_{\text {rot }}$ & References \\
\hline SDSS J224741.46+145638.8 & 18000 & $\mathrm{H}$ & 42.11 & $\ldots$ & Külebi et al. $(08)$ \\
SDSS J225726.05+075541.7 & 40000 & $\mathrm{H}$ & 16.17 & $\ldots$ & Külebi et al. $(08)$ \\
KUV 813-14 & 11000 & $\mathrm{H}$ & 45 & $18 \mathrm{~d}$ & \\
SDSS J231951.73+010909.3 & 8300 & $\mathrm{H}$ & 9.35 & $\ldots$ & Külebi et al. $(08)$ \\
SDSS J232248.22+003900.9 & 39000 & $\mathrm{H}$ & 21.40 & $\ldots$ & Külebi et al. $(08)$ \\
SDSS J232337.55-004628.2 & 15000 & $\mathrm{He}$ & 4.80 & $\ldots$ & \\
PG 2329+267 & 9400 & $\mathrm{H}$ & 2.31 & $\ldots$ & \\
SDSS J234623.69-102357.0 & 8500 & $\mathrm{H}$ & 9.17 & $\ldots$ & Külebi et al. $(08)$ \\
SDSS J234605.44+385337.7 & 26000 & $\mathrm{H}$ & 1000.00 & $\ldots$ & \\
LTT 9857 & 8570 & $\mathrm{H}$ & 0.0031 & $\ldots$ & \\
& & & & & \\
\hline
\end{tabular}

\section{Modelling spectra and polarisation of magnetic white dwarfs}

These identifications with stationary line components allowed an estimation of the approximate range of field strengths covering the stellar surface. However, the detailed field structure could not be inferred. This was only possible by simulating the radiative transfer through a magnetized stellar atmospheres, as e.g. described by Jordan (1992). In order to calculate theoretical flux and polarisation spectra for a given geometry of the magnetic field, theoretical Stokes parameters have to be evaluated on a few hundred surface elements taking into account the local magnetic field strength and the orientation of the magnetic field with respect to the observer and the normal of the stellar surface. Afterward, the results from the respective surface elements must be summed up.

Such a forward calculation for a given magnetic field geometry is relatively simple. The goal, however, is to determine the magnetic field geometry from the observed spectra and polarisation data. Since it turned out that most magnetic white dwarfs could not be described by simple dipole fields (see eg. Achilleos \& Wickramasinghe 1989), the number of free parameters to describe the field geometry can be very large. Therefore, finding a magnetic field geometry that adequately reproduces the observed spectro-polarimetric data is extremely tedious and practically impossible when time resolved observations of rotating magnetic white dwarfs are used that have to reproduce the observation for all different rotational phases.

For the first time Burleigh et al. (1999) used an automatic least-squares fit procedure to determine the magnetic field geometry of RE J 0317-853 from phase resolved HST data (see Fig. 3) They used a downhill simplex method in multidimensions to account for the rotational geometry and an assumed offset-dipole configuration of the magnetic field. However, the approach has the disadvantage of easily running into local minima rather than the global $\chi^{2}$ minimum of the high-dimensional parameter space. It also turned out that the optical spectro-polarimetric data of RE J 0317-853 are not fully consistent with the geometry derived from the UV data so that more sophisticated fit procedures need to be used.

A rather successful approach is the application of genetic (Jordan) and evolutionary (Euchner) algorithms to find a magnetic field geometry that is consistent with the spectropolarimetric data of for all rotational phases (Euchner et al. 2002; Gänsicke et al. 2002; Euchner et al. 2005, 2006; Beuermann et al. 2007). 

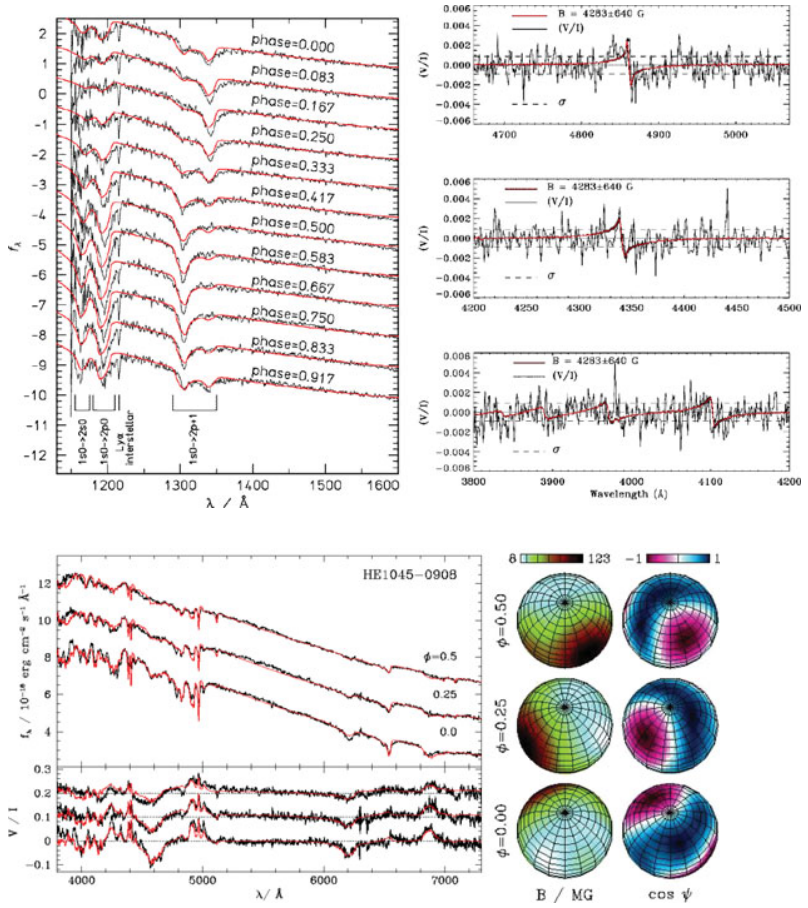

Figure 2. Left side: Observed and theoretical spectra UV spectra for 12 different phase across the $725 \mathrm{~s}$ rotation period of RE J 0317-853 for a magnetic dipole with a polar field strength of $363 \mathrm{MG}$, shifted by 0.19 stellar radii along the dipole axis (Burleigh et al. 1999). Right: Measurement of a $4.3 \mathrm{kG}$ magnetic field in the white dwarf WD 446-789.

\section{Chemical species other than hydrogen}

All really detailed analyses of magnetic white dwarfs were limited to those with hydrogen-rich atmospheres. The reason is that atomic data for helium have not consistently been included into our radiative transfer models yet. First tentative analyses using the new helium data were, however, performed by Wickramasinghe et al. (2002).

In some cool $\left(T_{\text {eff }}<9000 \mathrm{~K}\right)$ helium rich magnetic white dwarfs, carbon molecules $\left(\mathrm{C}_{2}\right.$, $\mathrm{CH})$ are responsible for the absorption. Bues \& Pragal (1989) have interpreted such spectra with very simple approximations of the molecular physics of the Swan bands of $\mathrm{C}_{2}$ in magnetic fields between 10 and $150 \mathrm{MG}$. Berdyugina et al. (2007) has used a Paschen-Back approximation for the $\mathrm{C}_{2}$ absorption but has not yet analyse such objects with state-of-the-art radiation transfer models.

One of the most exciting recent discoveries is the detection of non-radial pulsations in a magnetic white dwarf with a hot carbon-rich atmosphere (Dufour et al. 2008).

\section{Magnetic white dwarfs with high magnetic fields}

About $10 \%$ of all isolated white dwarfs posses surface magnetic fields of more that $10^{6} \mathrm{G}$ (Liebert J. et al. 2005). Generally, magnetic white dwarfs with MG fields tend to be more massive than non-magnetic ones (Liebert J. 1988), but the number of objects with reliable mass determinations is rather small.

The Sloan Digital Sky Survey (SDSS) has almost tripled the number of known white dwarfs with MG fields (Schmidt et al. 2003; Vanlandingham et al. 2005). Külebi et al. (2008) have analysed all hydrogen-rich magnetic white dwarfs and found that almost all objects show indications field geometries which are more complicated than centered magnetic dipoles (see also Külebi et al. in these proceedings). The list of all currently know magnetic white dwarfs is provided in Table. 1. 


\section{Kilogauss magnetic fields in white dwarfs and their progenitors}

Until recently, magnetic fields below $30 \mathrm{kG}$ could not be detected, with the exception of the very bright white dwarf 40 Eri B $(V=8.5)$, in which Fabrika et al. (2003) found a magnetic field of $4 \mathrm{kG}$. However, by using the ESO VLT, we could push the detection limit down to about $1 \mathrm{kG}$ in two investigations of $22 \mathrm{DA}$ white dwarfs with $11<V<14$ (Aznar Cuadrado et al. 2004; Jordan et al. 2007) by means of polarimetry. The degree of polarization in the vicinity of the spectral lines caused by $1 \mathrm{kG}$ is only about $0.1 \%$ (see Fig. 3 as an example).

Magnetic fields of kilogauss strength have also been detected in the direct progeny of white dwarfs - central stars of planetary nebulae Jordan et al. (2005), probably the channel leading to about $99 \%$ of all white dwarfs, and hot subdwarfs O'Toole et al. (2005), accounting for about 1\%. The magnetic fields found were between 1 and $3 \mathrm{kG}$. If the magnetic flux is fully conserved during the transition phase to white dwarfs - these objects will become magnetic white dwarfs with fields up to $2 \mathrm{MG}$.

However, great care is necessary, since these detections in central stars and subdwarfs are at the very limit of what is observationally possible. Together with new observations of central stars of planetary nebular we are also checking the data analysis again in order to be convinced that the detections are real.

\section{Origin of magnetic white dwarfs}

The strong magnetic fields can be fossil if the magnetic flux is conserved during stellar evolution. The very high electric conductivity of degenerate electron gas in the interior of white dwarfs leads to very long decay times ( $\gtrsim 10^{10} \mathrm{yr}$ ). For a long time it was assumed that the higher modes decay more rapidly than the fundamental (Chanmugam et al. 1972; Fontaine et al. 1973). However, Muslimov et al. (1995) have shown that a weak quadrupole (or octupole, etc.) component on the surface magnetic field of a white dwarf may survive the dipole component and specific initial conditions: Particularly the evolution of the quadrupole mode is very sensitive (via Hall effect) to the presence of internal toroidal field. Under certain conditions, the higher-order components of the magnetic field my survive as long as the dipole component.

This shows that, in principle, higher-order multipoles may give us some information about internal magnetization of white dwarfs and the initial conditions from the prewhite dwarf evolution. Therefore, further investigations of the complex magnetic fields of white dwarfs remain important.

The magnetic fluxes of the main-sequence stars with the highest magnetic fields are very similar to the highest magnetic fields found in magnetic white dwarfs $\left(10^{9} \mathrm{G}\right)$. However, it is not easy to identify the main-sequence progenitors if we assume the fossil-field hypothesis, because magnetic fields below a few hundred Gauss are currently not detectable; moreover, magnetic fields in later type do not contain large-scale magnetic fields so that they also escape detection. The situation is also complicated by observational biases in the selection of the magnetic white dwarfs themselves. By applying initial-final mass relations and studying the number statistics Wickramasinghe \& Ferrario (2005) concluded that magnetic white dwarfs cannot come from $A_{p}$ and $B_{p}$ stars alone.

\section{Acknowledgements}

I thank Baybars Külebi for his help preparing Table 1. The work on magnetic white dwarfs was supported by DLR project 50 OR 0802 . 


\section{References}

Achilleos, N. \& Wickramasinghe, D. T. 1989, ApJ 346, 444

Angel J. R. P. 1979, in White Dwarfs and Variable Deg. Stars, Univ. of Rochester Press, p.313

Aznar Cuadrado, R., Jordan, S., Napiwotzki, R., Schmid, H. M., Solanki, S. K., \& Mathys, G. 2004, A\&SA 423, 1081

Barstow, M. A., Jordan, S., O'Donoghue, D., Burleigh, M. R., Napiwotzki, R., \& Harrop-Allin, M. K. 1995, MNRAS 277, 971

Berdyugina, S. V., Berdyugin, A. V., \& Piirola, V. 2007, Phys. Rev. Lett. 99, 1101

Berdyugin, A. V. \& Piirola, V. 1999, A\&A 352, 619

Beuermann K., Euchner F., Reinsch K., Jordan S., \& Gänsicke B. T. 2007, A\&A 463, 647

Blackett P. M. S. 1947, Nature 159, 658

Bues, I. \& Pragal, M. 1989, in White Dwarfs, Lecture Notes in Physics 328, ed. G. Wegner, p. 329

Burleigh, M. R., Jordan, S., \& Schweizer, W. 1999, ApJ 510, L37

Chanmugam G. \& Gabriel M. 1972, A\&A 16, 149

Dufour, P., Fontain, G., Liebert, J., Williams, K., \& Lai, D. K. 2008, ApJ 683, 167

Euchner F., Jordan S., Beuermann K., Gänsicke B., \& Hessman F. V. 2002, A 6 A 390, 633

Euchner, F., Reinsch, K., Jordan, S., Beuermann, K., \& Gänsicke, B. T. 2005, A\& $A$ 442, 651

Euchner, F., Jordan, S., Beuermann, K., Reinsch, K., \& Gänsicke, B. T. 2006 A\&A 451, 671

Fabrika S. N., Valyavin G. G., \& Burlakova T. E. 2003, Astronomy Letters 29, 737

Fontaine G., Thomas J. H., \& Van Horn H. M. 1973, ApJ 184, 911

Forster H., Strupat W., Rösner W., Wunner G., Ruder H., \& Herold H. 1984, J.Phys. V 17, 1301

Garstang R. H. 1977, Rep.Prog.Phys. 40, 105

Gänsicke, B. T., Euchner, F., \& Jordan, S. 2002, A\&A 394, 957

Ginzburg V. L. 1964, Sov. Phys. Dokl. 9, 329

Greenstein J. L. 1984, ApJ 281, L47

Greenstein J. L., Henry R. J. W., \& O'Connell R. F. 1985, ApJ 289, L25

Henry R. J. W. \& O'Connell R. F. 1984, ApJ 282, L97

Jordan, S. 1992, $A \& A, 265,570$

Jordan, S., Schmelcher, P., \& Becken W. 1998, A\&SA, 336, 33

Jordan, S., Schmelcher, P., Becken W., \& Schweizer, W. 2001, A\&A 376, 614

Jordan, S., Werner, K., \& O'Toole, S. J. 2005, A\& A 432, 273

Jordan, S., Aznar Cuadrado, R., Napiwotzki, R., Schmid, H. M., \& Solanki, S. K. 2007, A\&A 462,1101

Karl, C. A., Napiwotzki, R., Heber, U., Dreizler, S., Koester, D., \& Reid, I. N. 2005, A\&্A 434, 637

Kawka, A, Vennes, S, Schmidt G. D., Wickramasinghe D. T., \& Koch R. 2007, ApJ 654, 499

Kemp J. C. 1970, ApJ 162, 169

Kemic S. B. 1974a, ApJ 193, 213

Kemic S. B. 1974b, JILA Rep. 133

Külebi, B., Jordan, S., Euchner F., Hirsch, H., \& Löffler, W. 2008, Proceedings of the 16th European Workshop on White Dwarfs, Barcelona, in press

Liebert J. 1988, PASP 100, 1302

Liebert J. et al. 2005, AJ 129, 2376

Muslimov A. G., Van Horn H. M., \& Wood M. A. 1995, ApJ 442, 758

O'Toole, S. J., Jordan, S., Friedrich, S., \& Heber, U. 2005, A $\& A$ 437, 227

Preston G. W. 1970, ApJ 160, L143

Reimers, D., Jordan, S., \& Christlieb, N. 2004, A\&A 414, 1105

Rösner W., Wunner G., Herold H., \& Ruder H. 1984, J.Phys. V 17, 29

Schmidt, G. D. et al. 2003, ApJ 595, 1101

Vanlandingham, K. M. et al. 2005, AJ 130, 734

Wickramasinghe, D. T., Schmidt, G. D., Ferrario, L., \& Vennes, S. 2002, MNRAS 332, 29

Wickramasinghe, D. T. \& Ferrario, L. 2005, MNRAS 356, 1576

Woltjer L. 1964, ApJ 140, 1309 